\title{
Covid-19 and Ear Surgery: Treatment Strategies and Triage during the Post-lockdown Period
}

\author{
Rohit Bhardwaj $^{1} \cdot$ Akriti Sharma $^{2}$ (1) $\cdot$ Bhumika Singhal $^{2}$
}

Received: 12 December 2020/ Accepted: 22 March 2021 / Published online: 26 March 2021

(C) Association of Otolaryngologists of India 2021

\begin{abstract}
The accumulated disease burden during lockdown period, due to postponement of regular surgeries, is expected to put additional pressure on surgeons during the post-lockdown period. Due to the contagious nature of SARS-CoV-2 and its suspected presence in middle ear mucosa and mastoid, Ear surgeons are bound to face a challenging situation in present times as well as in times to come. Through this article we aim to streamline fresh management strategies particularly for the post-lockdown period keeping in mind that immunity after vaccination may take a few months to develop due to various factors discussed in the article. The ENT Cochrane, Pubmed and Web of Science databases were searched extensively using the terms 'Covid-19 and SARS-CoV-2 in conjunction with Ear surgery and Otology. Data collected from these, put together with our experience helped us in putting forward fresh management strategies to deal with the current situation being experienced worldwide. To reduce the risk of infection to the healthcare staff, we have suggested a new triage strategy for ear surgeries to reduce the accumulated disease burden in the post-lockdown period until immunity by vaccination develops amongst a good number of Ear surgeons. Also we have put forward certain operating guidelines that might prove helpful for the Ear surgeon during these times. Fresh Triage guidelines mentioned in
\end{abstract}

Akriti Sharma

drakriti2709@gmail.com

1 Department of Otorhinolaryngology, Safdarjung Hospital and Vardhman Mahavir Medical College, Ansari Nagar, Delhi, New Delhi 110029, India

2 Department of Otorhinolaryngology, SGT Medical College, Hospital \& Research Institute, Village Budhera, Gurugram, Haryana 122505, India this article are particularly meant to help ear surgeons reduce the accumulated disease burden in the post lockdown-period with ease and efficacy. Since ear surgery poses a risk of infection to the healthcare workers, specific guidelines pertaining to ear surgery during the pandemic are mentioned in detail which in our opinion can be of immense help to all the healthcare professionals involved in ear procedures till the time the vaccine is administered on a large scale.

Keywords Ear surgery · Covid-19 · Triage · Precautions · Post lockdown

\section{Introduction}

Covid-19 was declared a public health emergency by the World Health Organisationon 30 Jan 2020 [1]. It was declared a pandemic on 11 March 2020. Due to the highly contagious characteristic of SARS CoV-2, lockdowns were implemented worldwide. People were advised to avoid hospital visits for conditionsthat were non-life threatening or those that could be dealt with by online consultation. This eventually resulted in accumulation of disease burden over a period of time, particularly for surgical specialties. In the present scenario, neither is a definitive cure with worldwide consensus available for the disease nor does one see the light of the day with an effective vaccine being available worldwide despite various efforts. With increasing number of patients returning to hospitals for surgical intervention, surgeons are bound to face a challenging situation. This will put health care professionals at a high risk of acquiring infection due to close contact with confirmed or suspected cases of Covid-19 [2]. 
Although in the present scenario, there are different vaccines being made available against COVID 19 worldwide. As per WHO there are almost 66 vaccines in clinical trial phase and 176 in pre-clinical trial phase [3]. However it will still take few months to have a complete control over the number of Covid-19 cases, since most of the vaccines will require administration of two or more doses, at least 4 weeks apart to induce sufficient immune response. The immunity given by these vaccines is also not very long lasting. Few of the vaccines are still under trial phase and have been allowed "accelerated" authorization for use of drugs after the second phase of trials for "unmet medical needs of serious and life-threatening diseases in the countries".

In such circumstances, the production, availability and cost effectivity of these vaccines are other challenges that are being encountered. Many countries are still in the process of importing these vaccines. Another factor to take into account is that vaccines are not freely available for the general population and goverments in different nations are prioritizing the subgroup of populations (like healthcare workers) to be vaccinated first. Moreover, a lot of resistance is being seen amongst healthcare workers in volunteering to take the vaccine. Keeping all these aspects in mind, we still believe that triage of cases and appropriate precautions among ear surgeons, should still be continued for the coming months till the number of cases show a massive decline and the vaccination drive picks up.

As SARS CoV-2 is known to reside in very high concentrations in respiratory mucosa (nasal, nasopharyngeal and lower airways), and transmission mostly occurs via droplet spreads or aerosolized particles from infected epithelium or mucosa, any surgical intervention involving respiratory epithelium is a significant risk factor for disease transmission [4]. Hence, the risk for otologists performing ear surgery would be significant as the middle ear and mastoid mucosa is an embryological extension of respiratory epithelium (supported by histological analysis of both) and is in continuity with it. Moreover, there are reports suggesting presence of various respiratory viruses like rhinovirus, respiratory syncytial virus and corona viruses in cases of otitis media $[5,6]$.

Thus taking into consideration the available literature on ear surgery during the Covid-19 Pandemic, along with our experience, we aim to share certain guidelines and triage strategies which in our opinion, would be of great benefit to Ear surgeons in the present scenario.

\section{Methods}

The ENT Cochrane, Pubmed and Web of Science databases were searched using the terms 'Covid-19' and 'SARS-CoV-2' in conjunction with 'Ear surgery' and 'Otology'. The databases focused on the newest reports using these terms.

\section{Triage during the Post-lockdown Phase}

The possible presence of corona virus in middle ear mucosa (either due to continuous spread from respiratory epithelium or via blood transmission) has already been shown, hence there are definitely increased chances of contamination and spread of infection during otological procedures or surgeries $[7,8]$. The risk of contamination is further increased by generation of aerosols in operating theater if high speed surgical drills are in use during procedures $[9,10]$. This puts everyone in the surgical team at an increased risk of getting infected.

For providing continuous quality services in the field of Otology the healthcare workers in surgical team should protect themselves from exposure but before that one must device an appropriate triage strategy particularly for the post-lockdown period.

It is important to understand that, while there would be an additional pressure on surgeons during this time, due to an accumulated disease burden, not every case would demand prompt surgical intervention. In our opinion, a good triage strategy would be of immense help in effectively managing the disease burden during these times. Figure 1 depicts a triage strategy for ear surgery during the post-lockdown period in detail.

We believe that a case based approach is to be developed by the surgical team. It is the operating surgeon who must decide when a surgery needs to be undertaken keeping in mind the nature, stage and progression of the disease and above all the overall condition of the patient. The possible risks and benefits of surgery to the patient along with risks of exposure to the surgical team must be kept in mind.

Keeping these in mind, once the decision to go ahead with a surgery is made, following certain essential guidelines to minimize the risk of exposure to the healthcare workers as well as the patient is a must.

Certain generalized measures are advocated for the ear surgeon and his team to avoid getting infected during these unprecedented times. These guidelines and recommendations are based on our experience and recently published literature on Ear surgery in times of the COVID-19 pandemic. We have however written these particularly for the post-lockdown period as we believe that a specialized 
Fig. 1 Triage strategy for ear surgery during the postlockdown period

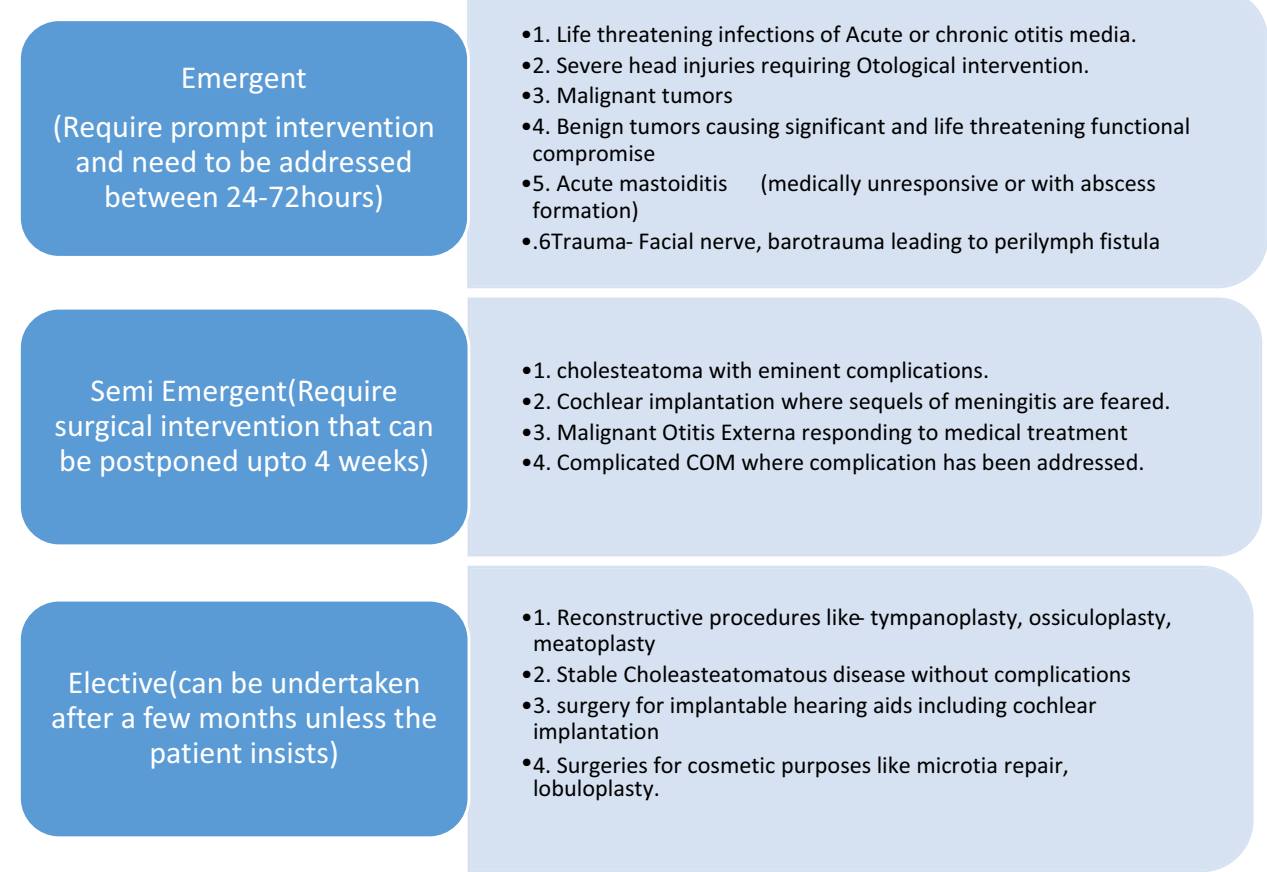

approach during this time will reduce the disease burden as well as the risk of infection to the healthcare staff while treating patients at the same time.

\section{Operating Guidelines for the Ear Surgeon during the Post-lockdown Period}

\section{Pre-operative Diagnostic Work up}

Most of the ear surgeries carry high risk of transmission of the SARS-Cov-2 virus by generating aerosols [11]. It is advised to assess the. Covid-19 status of every patient being prepared for surgery. In our opinion, RT-PCR must be done before any surgical procedure unless the case needs immediate intervention and the test cannot be done in that institute. Rapid antigen tests (although lacking desired specificity and sensitivity) should be considered in case of absolute emergencies. In cases where this is not possible the surgeon must take all precautions that are taken to for a covid-19 suspect. In cases of elective surgeries, patients could be asked to maintain a brief period of self-isolation before getting the test done for covid-19 screening prior to surgery. The guidelines given by the Royal College of Surgeons, England mention the need for isolating the patient 14 days prior to an elective surgery, and in cases where a patient belongs to the pediatrics age group, mention that one both parents must isolate with the child [12]. While a CT-Chest can be used to screen the patients who are symptomatic suspects of Covid-19 having negative RT-PCR results; screening of asymptomatic individuals is not supported by existing literature [13]. This also holds more importance when the patients are to undergo mechanical ventilation for general anesthesia for surgery because role mechanical ventilation in covid patients is not uniformly agreed upon, having both positive and negative outcomes [14].

Out of the 22 patients undergoing ear surgery at our centre during these times, 6 were emergency surgeries and were carried out in the emergency OT on the same day. These included 2 cases of post-auricular abscess requiring incision, drainage and mastoid exploration, one was a case of traumatic facial palsy needing facial nerve decompression, another one was a case of cholesteatoma with labyrinthitis as its complication and 2 were cases of impacted foreign body of the ear requiring their removal under general anaesthesia. The cases were carried out after the rapid antigen test report was found to be negative for all the patients, and their relatives. In spite of this report being negative, all precautions including wearing proper PPE, close fitting goggles, wearing double gloves and carrying out these procedures with trained and minimal number of healthcare workers were taken. The patients were considered Covid suspects and were kept in isolation till their RT-PCR report was awaited. The healthcare staff involved in OT with these patients, was also asked to remain quarantined for that particular period. The RT-PCR report of one of the six patients was found to be positive, though he had no symptoms of the disease. While 5 out of 6 patients with negative RT-PCR test were discharged $24 \mathrm{~h}$ after surgery and asked to quarantine themselves for a fortnight, 
one patient with a positive RT-PCR test was kept in an isolated room in the hospital for the next 14 days and went home after two RT-PCR test reports were found negative. The entire healthcare team including the anesthetists, surgeons and nursing staff present while operating upon this patient, along with one relative of the patient got themselves tested twice for covid-19 and remained quarantined for 14 days. All except one of their RT-PCR reports were found negative. One staff nurse who tested positive for covid-19 did not develop any symptoms in a span of 14 days and tested negative twice before she was declared disease free.

All of the 16 patients who underwent elective ear surgery at our centre, were first asked to isolate themselves at home for 14 days, and then admitted to the hospital after a negative rapid antigen test report, of the patients along with their attendants. Only one attendant was allowed for one patient and the RT-PCR sample was sent for both on the day of admission. Surgery was carried out only after RTPCR reports of all the patients and attendants were negative. The elective cases were mostly postponed cases of tympanoplasties and mastoidectomies that were worked up earlier and could not be performed due to lockdown. Eleven out of sixteen of these patients were operated under local anesthesia while five were operated upon under general anesthesia. While the patients operated upon in local anesthesia were discharged on the same day, those operated under GA were discharged after $24 \mathrm{~h}$.

All patients were asked to remain quarantined for 14 days at home and to report to their doctor for any Covid related symptoms (which were mentioned on their discharge cards) via teleconsultation.

\section{Organizing Surgical Team}

Ear surgeries being highly risky for the healthcare staff, need to be performed with minimum number of people in the OT. This will also reduce the number of PPE kits used during surgery which might also bring some economic relief. Also reducing the surgical time will further reduce this risk for the entire team. Thus we recommend that the most skilled surgeons should perform surgeries during these times. The efficient nursing and technical assistants who have worked previously with the operating surgeon should be involved. Team constitution should also consider involving those who are seropositive and have developed sufficient immunity by clinical or subclinical infections. All the members of surgical team must be trained in donning and doffing of PPEs, by organizing training sessions for the same.

For training purposes, the use of distal digital display systems or video recording would be a better option rather than direct involvement of trainees or post-graduate students in all surgeries, but since there is a need to impart practical hands on experience to the post graduates, it is suggested that a few elective surgeries could be performed by the post-graduates with consultant supervision, with timely completion of the procedure being made a priority [12].

\section{Preparing the Operation Theater}

The Operation Theater must be considered a "Highly contagious area" from the start of surgery till proper cleaning has been done after the procedure. In and out movements are to be restricted once the surgery starts and only permitted in very essential circumstances. Pictorial depiction or pasting of posters in the area marked for donning and doffing, to help team members, is suggested. Operation theaters having effective ventilation systems should only be used. The Royal College of Surgeons, England recommend the avoidance of fenestrated suction in cases where ear surgery is conducted on a patient with a wet ear, to minimize the risk of contamination by aerosols [12] They also recommend checking portable suction machines for unfiltered venting and adding extra filters to them if required. We believe that larger area theaters can also help, possibly by reducing concentration of contaminated aerosols per unit volume. For cleaning of operating tables and other furniture, standard disinfectants effective against micro-organisms must be used to minimize hand contamination and virus transmission [15]. The use of standard five gear Personal protective equipment including N-95 or PAPR masks for respiratory protection, gowns (long sleeve impervious) for body protection, protective goggles for eye protection, Face shield for face protection and gloves for hand protection is advocated. The importance of proper eye protection while performing ear surgery cannot be undermined, and while studies recommend (12) the use of Bolle tracker glasses, we believe that if they are not available, any protective glasses that fit close to the eye must be used. Standard guidelines with local amendments as per the hospital waste disposal committee must be set and followed for waste disposal.

\section{Anesthetic Considerations}

Prior to intubation, masks should be worn in a tight fitting manner to avoid air leak and thus aerosol contamination [16].

The guidelines put forward by Indian Academy of Otolaryngology and Head and Neck Surgery recommend that the patient must enter the OT wearing a surgical mask and pre-oxygenation must be done with the surgical mask. They also advocate the use of plastic drapes and hoods over 
patient's head with access holes during the process of preoxygenation and intubation of the patient [17].

In addition to these, we also recommend the use of video laryngoscopy over direct laryngoscopy for intubation as it would limit the risk of infection by avoiding close contact between anesthetist and the patient. While the intubation process is in progress the surgical team is advised to remain situated in the same OT with social distancing, and once surgical team takes over the anesthetists should monitor the patient with the same distance. Special care must be taken to avoid or minimize the lung trauma associated with mechanical ventilation during these times. Ventilation can be stopped just before extubation in order to generate fewer aerosols. Other significant considerations, mentioned in recent studies [18] must betaken into account by the anesthetist in order to contain the spread of infections.

\section{Surgical Considerations}

With respect to ear surgery, the following surgical considerations are to be kept in mind:

i) Microscope Vs Endoscope Microscope while having the advantage of offering dexterity and binocular vision on one hand, restricts the use of standard eye and face protection on other. While performing ear surgery, Eye protection is of paramount importance and while various suggestions for it have been published in literature, no ideal solution has been found yet. [19].For the same reason, using the microscope might pose a risk of face and eye contamination. In comparison to this, the endoscope would be better suited to offer face and eye protection or atleast prevent face and eye contamination. However, this would depend on the operating surgeon's skill and comfort as well. A well known method, also adopted by us, while operating during these times, is to operate under plastic drapes.

ii) Use of high speed surgical drills High speed drills have high probability of generating contaminated aerosols [8, 9]. Hence it is suggested that their use be restricted However when the use of drill is mandatory it must be used at a low speed to reduce generation of aerosols. The surgeon must also explore the possibility of limited damage control surgery/ multistep surgery instead of complete surgery considering the patient profile and disease course, to limit the uses of drills.

iii) Suction irrigation system Irrigation system should be titrated according to the need of drilling speed and burr type so as to optimize the aerosol generation, less irrigation will produce aerosols with bone particles and excessive irrigation will cause greater aerosol generation. Along with effective suction, a strong suction apparatus will further reduce the risk of generation of high volume contaminating aerosols.

\section{Post-operative follow up Considerations}

To further reduce the risk of cross infection between the patients and health care providers, minimum hospital stay and early discharge of patients is recommended. Use of absorbable sutures, Pre-operative patient counseling regarding the post-operative course and management plan and along with hard and soft copies of the same is suggested. Limited post-operative visits along with tele-consultation if required, is advocated.

\section{Conclusion}

Despite the various measures implemented worldwide to contain the spread of SARS-Cov-2, Covid-19 appears like it is here to stay for long or atleast till the successful distribution of effective vaccines on a large scale. As ear surgery seems to be a potential source of infection for the health care workers, we believe that triage of cases with ear disease requiring surgical intervention is still a must until vaccines are made easily available to both, the general population and healthcare workers on a large scale. Moreover, owing to the additional burden of elective and non-urgent cases needing surgical intervention in the current scenario, ear surgery needs to be undertaken taking all precautions such as wearing appropriate PPE with proper eye protection, N95 masks or PAPRs, minimizing the use of high speed drills, ensuring minimal days of patient stay in the hospital along with limited follow up visits and engaging the most experienced surgeons for surgery to minimize the number of time taken in the OT along with limiting the risk of infection to healthcare staff. We believe that these precautions must still be kept in mind till vaccines are easily available across the world and prove to be successful in controlling the spread of Covid-19.

\section{Declarations}

Conflicts of interests The authors declare that they have no conflicts of interests.

Ethical Approval Approval from the ethical committee was not required for this study. I confirm that the manuscript has not been published or submitted for publication to any other journal. 


\section{References}

1. Advice for the public on COVID-19-World Health Organization. Who.int. https://www.who.int/emergencies/diseases/novel-coro navirus-2019/advice-for-public. Published 2020. Accessed August 7, 2020.

2. Givi B, Schiff B, Chinn S et al (2020) Safety recommendations for evaluation and surgery of the head and neck during the covid19 pandemic. JAMA Otolaryngol-Head Neck Surg 146(6):579. https://doi.org/10.1001/jamaoto.2020.0780

3. Draft landscape and tracker of COVID-19 candidate vaccines. Who.int. https://www.who.int/publications/m/item/draft-landsca pe-of-covid-19-candidate-vaccines. Published 2021. Accessed February 14, 2021.

4. Patel ZM, Fernandez-Miranda J, Hwang PH et al (2020) Letter: precautions for endoscopic transnasal skull base surgery during the covid-19 pandemic. Neurosurgery 87(1):E66-E67. https://doi.org/10.1093/neuros/nyaa125

5. Pitkäranta A, Jero J, Arruda E, Virolainen A, Hayden F (1998) Polymerase chain reaction-based detection of rhinovirus, respiratory syncytial virus, and coronavirus in otitis media with effusion. J Pediatr 133(3):390-394. https://doi.org/10.10 16/s0022-3476(98)70276-8

6. Pitkäranta A, Virolainen A, Jero J, Arruda E, Hayden F (1998) Detection of rhinovirus, respiratory syncytial virus, and coronavirus infections in acute otitis media by reverse transcriptase polymerase chain reaction. Pediatrics 102(2):291-295. https://doi.org/10.1542/peds.102.2.291

7. Lavinsky J, Kosugi EM, Baptistella E et al (2020) An update on COVID-19 for the otorhinolaryngologist-a Brazilian association of otolaryngology and cervicofacial surgery (ABORL-CCF) position statement. Brazilian J Otorhinolaryngol 86(3):273-280

8. Heikkinen T, Thint M, Chonmaitree T (1999) Prevalence of various respiratory viruses in the middle ear during acute otitis media. N Engl J Med 340(4):260-264. https://doi.org/10.105 6/nejm199901283400402

9. Jewett D, Heinsohn P, Bennett C, Rosen A, Neuilly C (1992) Blood-containing aerosols generated by surgical techniques: a possible infectious hazard. AIHAJ 53(4):228-231. https://doi.org/ 10.1202/0002-8894(1992)053\%3c0228:bagbst\%3e2.0.co;2

10. Nokso-Koivisto J, Räty R, Blomqvist S et al (2003) Presence of specific viruses in the middle ear fluids and respiratory secretions of young children with acute otitis media. J Med Virol 72(2):241-248. https://doi.org/10.1002/jmv.10581

11. Tran K, Cimon K, Severn M, Pessoa-Silva C, Conly J (2012) Aerosol generating procedures and risk of transmission of acute respiratory infections to healthcare workers: a systematic review. PLoS ONE 7(4):e35797. https://doi.org/10.1371/journal.po ne.0035797

12. Otology: a graduated return to the provision of elective ENT services during the COVID-19 pandemic. Entuk.org. https://www.entuk.org/otology-graduated-return-provisionelective-ent-services-during-covid-19-pandemic. Published 2021. Accessed January 14, 2021.

13. Shao J, Ayuso S, Deerenberg E, Elhage S, Augenstein V, Heniford B (2020) A systematic review of CT chest in COVID-19 diagnosis and its potential application in a surgical setting. Colorectal Dis. https://doi.org/10.1111/codi.15252

14. Wunsch H (2020) Mechanical ventilation in covid-19: interpreting the current epidemiology. Am J RespirCrit Care Med 202(1):1-4. https://doi.org/10.1164/rccm.202004-1385ed

15. Réduction du risque de transmission du coronavirus SARS-CoV2 par la ventilation etgestion des effluents des patients. Hcsp.fr. https://www.hcsp.fr/Explore.cgi/

AvisRapportsDomaine?clefr=783. Published 2020. Accessed Augu Home. Iaohns.in. https://iaohns.in/home/. Published 2021. Accessed January 14, 2021 st 7, 2020.

16. https://sfar.org/principes-de-gestion-des-voies-aeriennes. (consulté le 07/04/2020)]. Published 2020. Accessed August 7, 2020.

17. .Home. Iaohns.in. https://iaohns.in/home/. Published 2021. Accessed January 14, 2021.

18. Gong Y, Cao X, Mei W et al.(2020) Anesthesia considerations and infection precautions for trauma and acute care cases during the COVID-19 pandemic. anesthesia \& analgesia. Publish ahead of print. doi:https://doi.org/10.1213/ane.0000000000004913.

19. Clamp P, Broomfield S (2020) The challenge of performing mastoidectomy using the operating microscope with coronavirus disease 2019 personal protective equipment (PPE). J Laryngol Otol 134(8):739-743. https://doi.org/10.1017/s002221512000 1607

Publisher's Note Springer Nature remains neutral with regard to jurisdictional claims in published maps and institutional affiliations. 